the danger of contamination and drowning in their own honeydew. These insects coat the honeydew with wax secreted from specialized glands ${ }^{3}$.

Furthermore, some gall-forming aphid species clean their galls by pushing the coated honeydew outside ${ }^{4-6}$. The coated elastic honeydew droplets can be pushed, rolled and squeezed without leaking or wetting. $\mathrm{Smith}^{3}$ noted that this behaviour was initially observed by Buckton in 1876 and may have promoted the evolution of social organization in aphids ${ }^{7}$. Practically, it is worthwhile examining whether aphid's wax is better at coating liquid droplets than are the spores of Lycopodium.

In short, 'liquid marbles' are yet another example of how insects 'developed' a technology through natural selection long before humans got around to it ${ }^{6}$. Technologists (high and low) and engineers should look for new solutions with the eyes of biologists.

M. Inbar ${ }^{\star}$, J. C. Schultz $\dagger$

${ }^{\star}$ Department of Biology, University of Haifa at Oranim, Tivon 36006, Israel

$\dagger$ Department of Entomology, Pennsylvania

State University, University Park,

Pennsylvannia 16802, USA

1. Aussillous, P. \& Quere, D. Nature 411, 924-927 (2001).

2. Mahadevan, L. Nature 411, 895-896 (2001).

3. Smith, R. G. Journal of Natural History 33, 513-530 (1999).

4. Aoki, S. Kontyû 48, 71-73 (1980).

5. Kurosu, U. \& Aoki, S. Journal of Ethology 9, 51-55 (1991).

6. Benton, T. G. \& Foster, W. A. Proceedings of the Royal Society of London 247, 199-202 (1992).

7. Stern, D. L. \& Foster, W. A. Biological Review 71, 27-79 (1996)

8. Akre, R. D. et al. Insects Did it First (Ye Galleon Press,

Washington, 1992).

\section{Disclosure of interests: there's a long way to go}

Sir - I read with great interest and approval your policy (Nature 412, 751; 2001) requiring authors to disclose financial interests. This policy has been sorely needed for a long time, and I hope that other journals follow suit. My only caveat is with respect to your anticipation that "employers" will "police" the policy.

I recently attended a symposium chaired by two key university counsel from two leading US universities (one private, one state), and asked them what they and their institutions do to ensure that scientists disclose financial interests in publishing articles. The answer was a blank stare - they assumed that such interests are disclosed as appropriate and have no procedures or policies for ensuring that such disclosures occur.

Tamsen Valoir

Jenkens \& Gilchrist, 1100 Louisiana Street, Suite

1800, Houston, Texas 77002, USA

\section{Sequenced strains must be saved from extinction}

Sir - Many prokaryotic strains used for genome sequencing projects are poorly documented and not generally available.

With improvements in sequencing technology and growing recognition of the value of microbial genome sequence data, the number of microbial genomesequencing projects is increasing rapidly. There are 56 completed prokaryotic genome sequences (10 strains of Archaea and 46 of Bacteria), and another 210 in progress (see, for example, www.tigr.org and www.integratedgenomics.com).

Of these 266 projects, some of which are performed on organisms not available in pure culture - some endosymbionts, for example - only 51 represent the type strain of the species. (A type strain is made up of living cultures of an organism descended from the nomenclatural type.) Of the rest, 138 represent non-type strains (a non-type strain is often selected only because it happens to be close at hand); 31 projects concern symbionts and environmental (uncultured) strains; 32 do not specify a strain; 14 represent prokaryotic species with invalid species names (validly named bacterial species are either on the 1980 approved lists of names, or validated after 1980 by taxonomic description in the International Journal of Systematic and Evolutionary Microbiology, or by validation in that journal: an invalid name has no standing in nomenclature and may be changed subsequently. Only 115 represent the type species of the genus (the typus of the genus included when the genus name was originally validly published) and only 123 are deposited in public culture collections. Mandatory deposition of the type strain of any validly described (culturable) prokaryotic species in a major public culture collection guarantees the availability of the strain and allows crossreferencing of published data.

When there were only a few projects, these taxonomic and preservation issues were not so evident. With the explosion in sequencing and the sequencing of multiple strains of a species (including Escherichia coli, Staphylococcus aureus and other major pathogens), questions of strain identity and safekeeping assume more importance. Deposition of strains in public collections with long-term funding is the only way to ensure their maintenance and their continuing availability to the scientific community. As things stand, it is a real possibility that a strain for which a wealth of genomic data has been generated may become "extinct" through loss of viability.

We propose that the following standards should be adopted by the entire community. First, genome-sequencing project lists and databases should include the name of the strain sequenced and its associated culture collection accession number(s), as well as its origin. Second, the type strain of a species should be used for sequencing unless other factors make this inappropriate. Third, strains for which genome sequences have been, or are being, generated should be deposited in at least two major public biological resource centres, such as the American Type Culture Collection, the German Collection of Microorganisms and Cell Cultures, the Pasteur Institute Collection or the Japanese Collection of Microorganisms. Naomi Ward ${ }^{\star}$, Jonathan Eisen ${ }^{\star}$, Claire Fraser ${ }^{\star}$, Erko Stackebrandt $\dagger$

${ }^{*}$ The Institute for Genomic Research, 9712 Medical Center Drive, Rockville, Maryland 20850, USA † German Collection of Microorganisms and Cell Cultures, Mascheroder Weg 1b, D-38124 Braunschweig, Germany

\section{Taxonomists make a name for themselves}

Sir - Many publications by taxonomists are not included in the Science Citation Index $(S C I)$, as pointed out in

Correspondence by A. G. Valdecasas et al. (Nature 403, 698; 2000) and by F. T. Krell (Nature 405, 507-508, 2000). E. Garfield (Nature 413, 107; 2001), on the other hand, pointed out some citation classics in taxonomy based on the SCI.

Your correspondents fail to mention the real reason most taxonomists are not included in the SCI: the way taxonomic research is cited means it does not feature in references. In referring to the name of a species, it is customary to state the name(s) of the author(s) after the species name, sometimes abbreviated and with the year of publication of the first description of the species. This citation method is ignored by the SCI and other citation indexes, which is why these scientists do not get the credit they deserve, even though each use of a species name in fact represents a citation.

A quick look in the ISI Web of Science 1988-2001 [www.isinet.com/isi/products/ citation/wos/index.html] shows that the most frequently cited scientists are the authors who named the bacterium Escherichia coli (108,262 citations), the yeast Saccharomyces cerevisiae $(43,403)$ and the fruitfly Drosophila melanogaster $(14,451)$. To put these numbers into perspective, Albert Einstein is cited 'only' 11,920 times.

\section{Gerard van der Velde}

Department of Animal Ecology and Ecophysiology, University of Nijmegen, Nijmegen, Toernooiveld 1, 6525 ED, The Netherlands 